\title{
PREVALENCE OF EXTENDED SPECTRUM BETA LACTAMASE (ESBL) PRODUCTION AMONG URINARY ISOLATES IN A TERTIARY CARE SETUP.....A BITTER TRUTH ABOUT THE SUPERBUGS....!!!!!!!!!
}

\author{
Nita Gangurde1, Preeti Bajaj², Rupali Pawar³, Varsha Sope
}

${ }^{1}$ Associate Professor, Department of Microbiology, Dr. Vasantrao Pawar Medical College, Hospital and Research Centre, Nashik, Maharashtra.

2Professor and Head, Department of Pathology, Dr. Vasantrao Pawar Medical College, Hospital and Research Centre, Nashik, Maharashtra.

${ }^{3}$ Assistant Lecturer, Department of Microbiology, Dr. Vasantrao Pawar Medical College, Hospital and Research Centre, Nashik, Maharashtra.

${ }^{4}$ Assistant Lecturer, Department of Microbiology, Dr. Vasantrao Pawar Medical College, Hospital and Research Centre, Nashik, Maharashtra.

\begin{abstract}
BACKGROUND

Urinary Tract Infection (UTI) occurs as a result of interaction between bacterial virulence and host biological and behavioural factors. Worldwide, about 150 million people are diagnosed with UTI each year, which costs the global economy. UTI although treatable, is now becoming increasingly tough to control, because of rampant antimicrobial resistance in the Enterobacteriaceae family, particularly in E. coli. ESBL (Extended Spectrum Beta Lactamase) producing organisms are distributed worldwide and their prevalence is increasing. Organisms responsible for UTI such as E. coli, Klebsiella species and Enterobacter species have the ability to produce ESBLs in large quantities. The World Health Organisation (WHO) has called antibiotic resistance an emerging disease. In almost all cases of UTI, empirical antimicrobial treatment initiated before the laboratory results of urine culture are available, thus, antibiotic resistance may increase in uropathogens due to frequent use of antibiotics. Therefore, the present study was conducted to study the bacterial spectrum causing UTI in our Institute and to find out the antibiotic response to these organisms with special emphasis on ESBL production by these organisms.

Aims and Objectives - 1) To study the bacterial spectrum causing UTI in our Institute; 2) To study the prevalence of drug resistance in Gram negative organisms to different urinary antibiotics with special emphasis on ESBL production.
\end{abstract}

\section{MATERIALS AND METHODS}

The prospective study was conducted over a period of three months (From October 2016 to December 2016) at Department of Microbiology, Dr. Vasantrao Pawar Medical College and Hospital, Nashik, Maharashtra, India. Total 955 urine samples received at Microbiology Laboratory were included in the study. Antibiotic Sensitivity Testing (AST) was performed using Kirby-Bauer Disc diffusion method on Mueller-Hinton agar by following the guidelines given by CLSI 2012. The antibiotic panel used for AST included - Gentamycin, Amikacin, Piperacillin, Cefotaxime, Cotrimoxazole, Ampicillin, Polymyxin B, Ceftazidime, Cefadroxil, Amoxiclav, Norfloxacin, Nitrofurantoin, Cefuroxime, Cefepime, Piperacillin-Tazobactam and Imipenem. All the isolates which showed resistance to third generation cephalosporins were further tested for confirmation of beta-lactamase production by phenotypic methods. Confirmation of ESBL production was done by using E-test (Hi-Media Laboratory, Mumbai).

Screening of ESBL production - This was done by two methods, a) Phenotypic Confirmatory Disc Diffusion Test (PCDDT); b) Double Disc Synergy Test (DDST).

Confirmation of ESBL production - This was done by using Ezy-MIC strips (Hi-Media Laboratories Pvt. Ltd.).

\section{RESULTS}

Total 955 urine samples were processed. Out of 955 samples, Gram Negative bacilli were isolated from 433 samples and Gram positive organisms were isolated from 54 samples. Out of 433 Gram negative organisms, 205 (47.3\%) were E. coli and 82 (18.9\%) were Klebsiella pneumonia, 50 (11.5\%) were Proteus species, $55(12.7 \%)$ were pseudomonas species and $41(9.4 \%)$ were Enterobacter species. Out of 433 Gram negative organisms, 158 were ESBL producers. Out of 158 ESBL producers E. coli were $45.5 \%$, Klebsiella pneumonia were $30.4 \%$, Proteus species were $24 \%$, Pseudomonas species were $25.45 \%$ and Enterobacter species were $31.7 \%$. Most of the organisms were resistant to third generation cephalosporins. ESBL producers showed resistance to Cefotaxime (81.01\%), Cefoperazone (82.2\%) and Ceftazidime (81.01\%). Resistance to aminoglycosides such as Amikacin (24.05\%) was found to be on lower side as compared to Gentamicin (62.02\%). Also resistance to Tetracycline was found to be on higher side (77.8\%). Sensitivity towards Piperacillin, Polymyxin B, Cefepime, Piperacillin + Tazobactam and Imipenem turned out to be satisfactory. E. coli shows higher percentage of resistance to third generation cephalosporins, Cefotaxime (85.1\%), Ceftazidime (88.2\%) and Cefoperazone (87.2\%). Klebsiella pneumoniae shows unsatisfactory results as compared to E. coli. Also, Klebsiella pneumoniae show higher amount of resistance to third generation cephalosporins such as Cefotaxime (96\%), Ceftazidime (96\%) and Cefoperazone (92\%). But resistance to higher antibiotics like Cefepime, Piperacillin + Tazobactam and Imipenem shows satisfactory level of sensitivity among all ESBL producers.

\section{CONCLUSION}

Increasing prevalence of ESBL producers among Gram negative organisms is nowadays becoming a great threat. This has become a major clinical problem in treating infections caused by ESBL producers. Increasing rate of resistance to commonly used antibiotics is an alarming sign for future of healthcare sector. Our study reveals that commonly used antibiotics are almost of no use for 
treatment of UTI patients. But special drugs like Cefepime, Piperacillin + Tazobactam and Imipenem could be kept as reserved drugs to treat infections. If not used rationally resistance to these reserved drugs will emerge, which will ultimately take us to situation like "NO ANTIBIOTIC ERA".....and which is a bitter truth about the Superbugs.....!!!

\section{KEYWORDS}

Urinary Tract Infections (UTI), ESBL, Drug Resistance.

HOW TO CITE THIS ARTICLE: Gangurde N, Bajaj P, Pawar R, et al. Prevalence of extended spectrum beta lactamase (ESBL) production among urinary isolates in a tertiary care setup.....a bitter truth about the superbugs....!!!!!!!!. J. Evolution Med. Dent. Sci. 2017;6(11):867-873, DOI: 10.14260/Jemds/2017/187

\section{BACKGROUND}

Urinary Tract Infection (UTI) occur as a result of interaction between bacterial virulence and host biologic and behavioural factors. " "UTI" is a broad term that encompasses either asymptomatic microbial colonisation of urine or asymptomatic infection with microbial invasion and inflammation of urinary tract.2,3 UTI remains the most common bacterial infection in human population and is one of the most frequently occurring nosocomial infections. 4,5 Worldwide, about 150 million people are diagnosed with UTI each year, which costs the global economy.6,7,8 Complicated UTIs are associated with comorbid conditions that prolong the need for treatment or increase the chances for therapeutic failure. ${ }^{9,10,11}$ UTI is presently the most commonly diagnosed infectious syndrome despite of the extensive availability of antimicrobial agents. So UTI although treatable, is now becoming increasingly tough to control because of rampant antimicrobial resistance in the Enterobacteriaceae family, particularly in E. coli. ${ }^{12,13,14}$

ESBL (Extended Spectrum Beta Lactamase) producing organisms are distributed worldwide and their prevalence is increasing. ${ }^{15}$ So Gram negative pathogens harbouring ESBLs have occurred numerous outbreaks of infections and are becoming an increasing therapeutic problem in many countries. ${ }^{16}$ Organisms responsible for UTI such as E. coli, Klebsiella species and Enterobacter Species have the ability to produce ESBLs in large quantities. These are enzymes capable of conferring bacterial resistance to the penicillins, first, second and third generation cephalosporins and aztreonam (but not the cephamycins or carbapenems) by hydrolysis of these antibiotics. ${ }^{13,17}$ But the plasmid responsible for ESBL production carry resistance to many antibiotics like aminoglycosides, fluoroquinolones, tetracyclines, chloramphenicol and cotrimoxazole.18,19,20 It has been found that with each new class of antibiotic, a new beta-lactamase emerged that caused resistance to that class of drug. Presumably, the selective pressure imposed by the use and overuse of new antibiotics has resulted in emergence of new variants of beta-lactamase. ${ }^{21}$

Majority of ESBL producing organisms are E. coli and Klebsiella pneumonia. The major risk factors implicated are

Financial or Other, Competing Interest: None.

Submission 19-01-2017, Peer Review 02-02-2017,

Acceptance 04-02-2017, Published 06-02-2017.

Corresponding Author:

Dr. Nita Gangurde,

\#C-501, Anmol Nayantara City One,

Near Durvankur Lawns, Bajirao Nagar,

Tidke Colony, Govind Nagar Link Road,

Nashik-422008, Maharashtra.

E-mail: nitagangurde@gmail.com

DOI: $10.14260 /$ jemds $/ 2017 / 187$

(c) $\underset{\mathrm{BY}}{\mathrm{NC}} \bigodot_{\mathrm{ND}}$ long-term exposure to antibiotics, prolonged ICU stay, nursing home residency, severe illness, etc. ${ }^{21,22}$ E. coli has been reported as the commonest isolate causing UTI, but few authors have reported changing pattern in prevalence of uropathogens. ${ }^{23,24,25}$ The World Health Organisation (WHO) has called antibiotic resistance an emerging disease. ${ }^{26}$ In almost all cases of UTI, empirical antimicrobial treatment initiates before the laboratory results of urine culture are available, thus antibiotic resistance may increase in uropathogens due to frequent use of antibiotics. 27,28 The prevalence of antimicrobial resistance in patients with UTI is increasing and can vary according to geographical and regional location. ${ }^{29}$

Therefore, the present study was conducted to study the bacterial spectrum causing UTI in our Institute and to find out the antibiotic response to these organisms with special emphasis on ESBL production by these organisms.

\section{Aims and Objectives}

- To study the bacterial spectrum causing UTI in our Institute.

- To study the prevalence of drug resistance in Gram negative organisms to different urinary antibiotics with special emphasis on ESBL production.

\section{MATERIALS AND METHODS}

The prospective study was conducted over a period of three months (From October 2016 to December 2016) at Department of Microbiology, Dr. Vasantrao Pawar Medical College and Hospital, Nashik, Maharashtra, India.

Total 955 urine samples received at Microbiology Laboratory were included in the study. The samples were inoculated on CLED (Cystine Lactose Electrolyte Deficiency) agar to study their cultural characteristics. Identification was done next day by using standard conventional, biochemical tests. Antibiotic Sensitivity Testing (AST) was performed using Kirby-Bauer Disc diffusion method on Mueller-Hinton agar by following the guidelines given by CLSI 2012. The antibiotic panel used for AST included - Gentamycin, Amikacin, Piperacillin, Cefotaxime, Cotrimoxazole, Ampicillin, Polymyxin B, Ceftazidime, Cefadroxil, Amoxyclav, Norfloxacin, Nitrofurantoin, Cefuroxime, Cefepime, PiperacillinTazobactam and Imipenem.

All the isolates which showed resistance to third generation cephalosporins were further tested for confirmation of beta-lactamase production by phenotypic methods. Confirmation of ESBL production was done by using E-test (Hi-Media Laboratory, Mumbai).

\section{Screening of ESBL Production}

This was done by two methods -

a) Phenotypic Confirmatory Disc Diffusion Test (PCDDT).

b) Double Disc Synergy Test (DDST). 
Phenotypic Confirmatory Disc Diffusion Test (PCDDT)

The suspected ESBL producing isolates were inoculated on Mueller-Hinton agar plates by lawn culture. Ceftazidime and Ceftazidime-Clavulanic acid discs were placed at a distance of $2.5 \mathrm{~cm}$ (centre to centre). Plates were incubated at $37^{\circ} \mathrm{C}$ overnight. Readings were taken next day. An increase in zone diameter for Ceftazidime-Clavulanic acid by $\geq 5 \mathrm{~mm}$ was considered positive for ESBL production (CLSI 2010).

\section{Double Disc Synergy Test (DDST)}

A single disc of Amoxyclav was placed at centre on MuellerHinton agar plate pre-swabbed with respective culture. Four antibiotics - Aztreonam, Cefotaxime, Ceftriaxone and Ceftriaxone discs were placed at a distance of $1.5 \mathrm{~cm}$ from centrally placed Amoxyclav disc. Plates were incubated at $37^{\circ} \mathrm{C}$ for 24 hours. Enhancement of zone of inhibition towards Clavulanic acid disc was considered indicative of ESBL producer. ${ }^{6}$

\section{Confirmation of ESBL Production}

This was done by using Ezy-MIC strips (Hi-Media Laboratories Pvt. Ltd.). These strips contained Ceftazidime on one side in two-fold gradient and Ceftazidime + Clavulanic acid combination on other side. The tests were done as per the manufacturer's instructions.

Reference strains used in the study were E. coli ATCC 25922 as negative control and Klebsiella pneumonia ATCC 700603 as positive control.

\section{RESULTS}

\begin{tabular}{|c|c|c|c|}
\hline \multirow{2}{*}{ Name of Organism } & $\mathbf{N = 4 3 3}$ & $\mathbf{N = 5 4}$ & \multirow{2}{*}{$\%$} \\
\cline { 2 - 3 } & $\begin{array}{c}\text { Gram } \\
\text { Negative }\end{array}$ & $\begin{array}{c}\text { Gram } \\
\text { Positive }\end{array}$ & \\
\hline E. coli & 205 & & 47.3 \\
\hline Klebsiella Species & 82 & & 18.9 \\
\hline Proteus Species & 55 & & 12.7 \\
\hline Pseudomonas Species & 50 & & 11.5 \\
\hline Enterobacter Species & 41 & & 9.4 \\
\hline Enterococcus Species & & 14 & 25.9 \\
\hline Staphylococcus aureus & & 40 & 74.0 \\
\hline $\begin{array}{c}\text { Total Gram Negative/ } \\
\text { Positive Organisms }\end{array}$ & 433 & 54 & \\
\hline Table 1. Spectrum of Organisms Isolated in Urine Samples \\
\hline
\end{tabular}

\begin{tabular}{|c|c|c|}
\hline $\begin{array}{c}\text { Name of } \\
\text { Organism (n) }\end{array}$ & $\begin{array}{c}\text { Number of } \\
\text { ESBL Producers }\end{array}$ & $\begin{array}{l}\% \text { of ESBL } \\
\text { Producers }\end{array}$ \\
\hline E. coli (205) & 94 & 45.5 \\
\hline Klebsiella Species (82) & 25 & 30.4 \\
\hline Proteus Species (55) & 12 & 24 \\
\hline Pseudomonas Species (50) & 14 & 25.45 \\
\hline Enterobacter Species (41) & 13 & 31.7 \\
\hline Total & 158 & \\
\hline \multicolumn{3}{|c|}{$\begin{array}{l}\text { Table 2. Incidence of ESBL Production among } \\
\text { Gram Negative Bacilli Isolated in Urine Samples }\end{array}$} \\
\hline
\end{tabular}

\begin{tabular}{|c|c|}
\hline $\begin{array}{c}\text { Name of } \\
\text { Antibiotic }\end{array}$ & $\begin{array}{c}\text { \% Resistance among } \\
\text { ESBL Producers }\end{array}$ \\
\hline Gentamicin & 62.02 \\
\hline Amikacin & 24.05 \\
\hline Ofloxacin & 74.05 \\
\hline Tetracycline & 77.8 \\
\hline Ciprofloxacin & 81.01 \\
\hline Chloramphenicol & 48.1 \\
\hline Piperacillin & 25.3 \\
\hline Cefotaxime & 81.01 \\
\hline Cotrimoxazole & 75.3 \\
\hline Ampicillin & 81.01 \\
\hline Polymyxin B & 15.1 \\
\hline Ceftazidime & 81.01 \\
\hline Cefoperazone & 82.2 \\
\hline Cefadroxil & 84.1 \\
\hline Amoxyclav & 48.1 \\
\hline Norfloxacin & 82.9 \\
\hline Nitrofurantoin & 41.1 \\
\hline Cefuroxime & 84.8 \\
\hline Cefepime & 10.1 \\
\hline Piperacillin + Tazobactam & 5.06 \\
\hline Imipenem & 3.1 \\
\hline Table 3. Antibiogram showing Resistance \\
Pattern of ESBL Producers regarding \\
different Antibiotics \\
\hline
\end{tabular}

\begin{tabular}{|c|c|c|c|c|c|c|c|c|c|c|c|}
\hline Organism & G & Ak & Of & Te & Cp & C & Pip & Ce & Co & A & Pb \\
\hline E. coli & 58.5 & 6.3 & 80.8 & 85.1 & 86.1 & 42.5 & 19.1 & 85.1 & 79.7 & 86.1 & 11.7 \\
\hline Klebsiella pneumoniae & 72 & 44 & 92 & 96 & 92 & 68 & 52 & 96 & 92 & 100 & 20 \\
\hline Proteus spp & 66.6 & 83.3 & 41.6 & 66.6 & 83.3 & 66.6 & 33.3 & 83.3 & 75 & 66.6 & 25 \\
\hline Pseudomonas spp & 57.1 & 28.5 & 57.1 & 57.1 & 64.2 & 50 & 14.2 & 78.5 & 50 & 50 & 0 \\
\hline Enterobacter spp & 69.2 & 30.7 & 38.4 & 23 & 38.4 & 30.7 & 23 & 23 & 38.4 & 53.8 & 38.4 \\
\hline
\end{tabular}

\begin{tabular}{|c|c|c|c|c|c|c|c|c|c|c|}
\hline Organism & Ca & Cs & Cfd & Ac & No & Nf & Cu & Cpm & Pt & I \\
\hline E. coli & 88.2 & 87.2 & 88.2 & 43.6 & 90.4 & 24.4 & 89.3 & 2.1 & 3.1 & 2.1 \\
\hline Klebsiella pneumoniae & 96 & 92 & 96 & 64 & 80 & 72 & 96 & 12 & 8 & 4 \\
\hline Proteus spp & 75 & 83.3 & 91.6 & 75 & 91.6 & 66.6 & 91.6 & 33.3 & 8.33 & 8.33 \\
\hline Pseudomonas spp & 64.2 & 64.2 & 50 & 42.8 & 64.2 & 57.1 & 57.1 & 21.4 & 7.1 & 0 \\
\hline Enterobacter spp & 23 & 46.1 & 61.5 & 30.7 & 46.1 & 61.5 & 53.8 & 30.7 & 7.6 & 7.6 \\
\hline
\end{tabular}

Table 4. Percent Resistance Pattern of ESBL Producers is Plated from Urine Samples with respect to Individual Antibiotics

G- Gentamicin, Ak- Amikacin, Of- Ofloxacin, Te- Tetracycline, Cp- Ciprofloxacin, C- Chloramphenicol, Pip- Piperacillin, CeCefotaxime, Co- Cotrimoxazole, A- Ampicillin, Pb- Polymyxin B, Ca- Ceftazidime, Cs- Cefoperazone, Cfd-Cefadroxil, Ac- Amoxicillin + clavulanic acid, No- Norfloxacin, Nf- Nitrofurantoin, $\mathrm{Cu}$ - Cefuroxime, Cpm- Cefepime, Pt- Piperacillin + Tazobactam, I- Imipenem 
Total 955 urine samples were processed. Out of 955 samples, Gram negative bacilli were isolated from 433 samples and Gram positive organisms isolated from 54 samples [Table 1].

Out of 433 Gram negative organisms 205 (47.3\%) were E. coli, 82 (18.9\%) were Klebsiella pneumonia, 50 (11.5\%) were Proteus species, 55 (12.7\%) were pseudomonas species and $41(9.4 \%)$ were Enterobacter species [Table 1]. Out of 433 Gram negative organisms, 158 were ESBL producers. Out of 158 ESBL producers E. coli were 45.5\%, Klebsiella pneumoniae were $30.4 \%$, Proteus species were $24 \%$, Pseudomonas species were $25.45 \%$ and Enterobacter species were $31.7 \%$ [Table 2].

Most of the organisms were resistant to third generation cephalosporins. ESBL producers showed resistance to Cefotaxime (81.01\%), Cefoperazone (82.2\%) and Ceftazidime $(81.01 \%)$ [Table 3]. Also, most of the ESBL producing organisms showed resistance to Fluoroquinolones, i.e. Ofloxacin (74.05\%), Ciprofloxacin (81.01\%) and Norfloxacin (82.9\%) [Table 3]. Ampicillin resistance was shown by $81.01 \%$ of ESBL producers [Table 3]. Resistance to aminoglycosides such as Amikacin $(24.05 \%)$ was found to be on lower side as compared to Gentamicin (62.02\%). Also resistance to Tetracycline was found to be on higher side (77.8\%) [Table 3]. Sensitivity towards Piperacillin, Polymyxin B, Cefepime, Piperacillin + Tazobactam and Imipenem turned out to be satisfactory [Table 3].

All ESBL producers were further confirmed for ESBL production by using Ezy-MIC strips. Out of 158 suspected ESBL producers, all were positive for confirmatory test for ESBL production.

E. coli shows higher percentage of resistance to third generation cephalosporins Cefotaxime (85.1\%), Ceftazidime $(88.2 \%)$ and Cefoperazone (87.2\%). Also, higher amount of resistance is shown by E. coli to Fluoroquinolones such as Ofloxacin (80.8\%) and Norfloxacin (90.4\%) [Table 4]. Sensitivity of $\mathrm{E}$. coli to Amikacin is quite satisfactory (Resistance 6.3\%).

Klebsiella pneumoniae shows unsatisfactory results as compared to E. coli. Also, Klebsiella pneumoniae show higher amount of resistance to third generation cephalosporins such as Cefotaxime (96\%), Ceftazidime (96\%) and Cefoperazone (92\%). Also, resistance to fluoroquinolones is much higher in percentage as compared to other organisms (Ofloxacin 92\% and Ciprofloxacin 92\%). Resistance to Tetracycline is also quite high (96\%) [Table 4]. Resistance to aminoglycoside is also on higher side in Klebsiella pneumoniae as compared to E. coli [Table 4]. Proteus species shows higher percentage of resistance to third generation cephalosporins and so to fluoroquinolones (Ciprofloxacin 83.3\% and Norfloxacin 80\%). In Pseudomonas species resistance to cephalosporins, aminoglycosides as well as fluoroquinolones was found to be on lower side as compared to other organisms. Enterobacter species was found to be least resistant organisms as compared to others. Resistance ratio to cephalosporins was low such as Cefotaxime was $23 \%$, Ceftazidime was $23 \%$ and Cefoperazone was $46.1 \%$. But Enterobacter species shows higher resistance to Gentamicin among all organisms (69.2\%) [Table 4].

But resistance to higher antibiotics like Cefepime, Piperacillin + Tazobactam and Imipenem shows satisfactory level of sensitivity among all ESBL producers [Table 4].

\section{DISCUSSION}

Increasing prevalence of ESBL producers among Gram negative organisms is nowadays becoming a great threat. This has become a major clinical problem in treating infections caused by ESBL producers. Resistance rates vary from country to country. 30 In present study among all uropathogens, E. coli is the predominant organism (47.3\%). Also among ESBL producers, E. coli is a dominant pathogen. Our finding is supported by findings from similar studies by $\mathrm{S}$. S. Tankhiwale et al who found E. coli as dominant uropathogen (49.8\%). ${ }^{31}$ Also Monsour Amin et al have reported E. coli as dominant uropathogens. ${ }^{32}$ Similar findings were reported by K. Aruna et al and other studies. ${ }^{33,34}$ A study from Vinita Dogra and B. A. Tatry et al shows same results. 35,36

Klebsiella pneumoniae were found to be second most common pathogen $(18.9 \%)$ in our study. And also second most common pathogen as ESBL producer in our study. Our this finding matches with findings from study by S. S. Tankhiwale who reported it as $37.8 \%{ }^{31}$ Monsour Amin et al reported it as $11.6 \% .^{32}$

In our study incidence of Klebsiella pneumoniae was followed by Proteus species (12.7\%), Pseudomonas species $(11.5 \%)$ and Enterobacter species (9.4\%). But percentage of ESBL production was higher among Enterobacter species. Our findings are similar to findings from study done by K. Aruna et al. ${ }^{6}$ Percentage of ESBL production in our study was found to be $24 \%$ in Proteus species, $25.45 \%$ in Pseudomonas species and $31.7 \%$ in Enterobacter species. Our findings go hand in hand with studies done by different people. ${ }^{37,38,39,40}$

As evident from results, the study demonstrates E. coli to be predominant organisms (47.3\%) amongst Gram negative organisms and Staphylococcus aureus (74\%) amongst Gram positive organisms. These findings are similar with other studies. ${ }^{41,42}$ In our study most of the isolates showed resistance to commonly used drugs like third generation cephalosporins and fluoroquinolones. These findings are similar with findings from other studies. ${ }^{43,44}$

In our study, we found Klebsiella pneumoniae as predominant drug resistant organism amongst all other organisms. This finding is contrary to findings from studies done by K. Aruna et al, ${ }^{6}$ study by Mohammad Akram ${ }^{7}$ and study by A. Bora $45,46,47$ who found E. coli as predominant drug resistant pathogen. But the results match with other similar studies. $31,48,49,50$ This could be due to difference in local prevalence of a particular organism.

It is also revealed that Ciprofloxacin and Norfloxacin, which are very commonly prescribed drugs for treatment of Urinary Tract Infections (UTI) are also showing higher percentage of resistance $(81.01 \%$ and $82.9 \%)$. These findings are similar with findings from $\mathrm{K}$. Aruna et $\mathrm{al}^{6}$ and $\mathrm{M}$. Eshwarappa et al. ${ }^{36}$

In present study, E. coli shows higher degree of resistance to third generation cephalosporins (almost above 80\%). This finding goes hand in hand with findings from Mohammad Akram et al. 7 But E. coli shows satisfactory degree of sensitivity to drugs like Cefepime $(2.1 \%$ resistance $)$ Piperacillin + Tazobactam (3.1\% resistance) and Imipenem (2.1\% resistance). This finding is similar with findings from study from Aligarh by Mohammad Akram et al. ${ }^{7}$ So it is evident that these drugs can be kept as reserved drugs to treat complicated UTIs.

Klebsiella pneumonia showed much higher degree of resistance to third generation cephalosporins and 
fluoroquinolones (above 90\%). But Klebsiella pneumonia shows satisfactory level of sensitivity to Cefepime (12\% resistance), Piperacillin + Tazobactam (8\% resistance) and Imipenem (4\% resistance). This is similar with study by Mohammad Akram. ${ }^{7}$ Satisfactory level of sensitivity to Carbapenems was also shown by other studies. 10,36

Other organisms like Proteus species, Pseudomonas species and Enterobacter Species showed higher degree of resistance to commonly used antibiotics. This is similar with other studies. ${ }^{51,52}$

Variations are seen in data from different locations. This could be due to drug prescribing trends present in those particular areas in same countries. Our country data may vary from data from other countries may be due to easy availability of antimicrobial drugs over the counter. 4

Now from above discussion, it is clear that antibiotic resistance is becoming a big problem for public health, which threatens the lives of hospitalised individuals as well as those with chronic conditions. ${ }^{7}$

Thus, in our study, resistance to third generation cephalosporins was found to coexist with resistance to two or more antibiotics like Ampicillin, Norfloxacin and Cotrimoxazole, etc. As also reported by Anbumani Narayanswamy, ${ }^{10}$ Subha et al ${ }^{51}$ and Duttatroy et al. ${ }^{53}$

\section{CONCLUSION}

Increasing rate of resistance to commonly used antibiotics is an alarming sign for future of healthcare sector. Injudicious use of antibiotics could be the most important reason for such high rate of increasing resistance. Our study reveals that commonly used antibiotics are almost of no use for treatment of UTI patients. But special drugs like Cefepime, Piperacillin + Tazobactam and Imipenem could be kept as reserved drugs to treat infections. But care should be taken that these drugs should not be used irrationally. These wonder drugs should be used judiciously for which the clinicians need to be made aware about the current scenario of resistance pattern regarding the multidrug resistant organisms. Otherwise, resistance to these reserved drugs will emerge which will ultimately take us to situation like "NO ANTIBIOTIC ERA".... and which is a bitter truth about the Superbugs.....!!!

\section{REFERENCES}

[1] Mandell G, Bennett J, Dolin R. Mandell, Douglas and Bennett's Principles and Practice of Infectiuos Diseases. 7th edn. Churchill Livingstone pp 876.

[2] Kumar Y, Sood S, Sharma A. Antibiogram and characterization of resistance markers among escherichia coli isolates from urinary tract infections. Journal Infect Dev Ctries 2013;7(7):513-9.

[3] Calvin MC. Urinary tract infections and pyelonephritis. In: Goldman L, Ausiella D. (eds). Cecil Textbook of Medicine. Philadelphia: Saunders Elsevier 2000:p 138.

[4] Nerurkar A, Solanky P, Naik SS. Bacterial pathogens in urinary tract infections and antibiotic susceptibility pattern. Journal of Pharmaceutical and Biomedical Sciences 2012;21(12):1-3.

[5] Gastmeier P, Kampf G, Wischnewski N, et al. Prevalence of nosocomial infections in representative German hospitals. J Hosp Infect 1998;38(1):37-49.
[6] Aruna K, Mobashshera T. Prevalence of extended spectrum beta lactamase production among uropathogens in South Mumbai and its antibiogram pattern. EXCLI J 2012;11:363-72.

[7] Akram M, Shahid M, Khan AU. Etiology and antibiotic resistance pattern of community acquired urianry tract infections in J N M C hospital, aligarh, India. Ann Clin Microbiol and Antimicrob 2007;6:4.

[8] Gozalez CM, Schaeffer AJ. Treatment of urinary tract infections: what's old, what's new and what works. World J Urol 1999;17(6):372-82.

[9] Rajan S, Prabhavathy JV. Antibiotic sensitivity and phenotypic detection of ESBL producing E.coli strains causing UTI in a community hospital, Chennai, Tamil Nadu, India. http://www.webmedcentral.com.

[10] Narayanswamy A, Mallika M. Prevalence and susceptibility of isolates of E.coli in a tertairy care hospital, Chennai-South India. IJM 2011;6(1):39-43.

[11] Lautenbach E, Patel JB, Bilker WB. Extended spectrum beta lactamase producing E.coli and klebsiella pneumonia, risk factors for infection and impact of resistance on outcomes. Clinical Infectious Diseases 2001;32(8):1162-71.

[12] Hussain A, Ewers C, Nandanwar N, et al. Multiresistant uropathogenic E.coli from a region in India where UTIs are endemic: genotypic \& phemotypic chracteristics of sequence type 131 isolates of the CTX-M-15 extended spectrum beta lactamase producing lineage. Antimicrob Agents Chemother 2012;56(12):6358-65.

[13] Paterson DL, Bonomo RA. Extended spectrum beta lactamase: a clinical update. Clin Microbial Rev 2005;18(4):657-86.

[14] Russo TA, Johnson JR. Medical and economic impact of extraintestinal infections due to E.coli: focus on an increasingly important endemic problem. Microbes Infect 2003;5(5):449-56.

[15] Afridi FI, Farooqi BJ, Hussain A. Frequency of extend. Sp. B. Lact. producing enterobacteriaceae among urinary pathogen isolates. Journal of the College of Physicians and Surgeons Pakistan 2011;21(12):741-4.

[16] Shobha KL, Rao GS, Rao S, et al. Prevalence of Ex. Sp. B. lact in urinary isolates of E.coli, klebsiella and citrobacter species and their antimicrobial susceptibility pattern in a tertiary care hospital. Indian Journal of Practising Doctors 2007;3(6):1-2.

[17] Metri BC, Jyothi P, Peerapur BV. Detection of ESBL in E.coli and K. pneumoniae isolated from urinary. Indian J Nephrol 2012;22(5):401-2.

[18] Naik J, Desai P. Antibiotic resistance pattern in urinary isolates of E.coli with special reference to extended spectrum beta-lactamses production. Int J of Pharm \& Life Sci 2012;3(3):1498-502.

[19] Jacoby GA, Sutton L. Properties of plasmids responsible for production of extended spectrum beta lactamases. Antimicrob Agents Chemother 1991;35(1):164-9.

[20] Nathisuwan S, Burgess DS, Lewis JS. ESBLs: epidemiology, detection and treatment. Pharmacotherapy 2001;21(8):920-8. 
[21] Asati RK. Antimicrobial sensitivity pattern of escherichia coli isolated from urine samples of UTI patients and issues related to the rational selection of antimicrobials. International Journal of Pharmacology and Therapeutics 2013;3(3):52-8.

[22] Kumar MS, Lakshmi V, Rajagopalan R. Occurence of extended spectrum beta lactamase among ebterobacteriaceae spp. isolated at a tertiary care institute. Indian J Med Microbiol 2006;24(3):208-11.

[23] Dash M, Padhi S, Mohanty I, et al. Antimicrobial resistance in pathogens causing urinary tract infections in a rural community of Odisha, India. Journal Family Community Med 2013;20(1):20-6.

[24] Omoregie R, Erebor JO, Ahonkahi I, et al. Observed changes in the prevalence of uropathogens in Benin city, Nigeria. NZJ Med Lab Sci 2008;62:29-31.

[25] Omoregie R, Eghafona NO. Urinary tract infection among asymptomatic HIV patients in Benin city, Nigeria. Br J Biomed Sci 2009;66(4):190-3.

[26] Mehta M, Bhardwaj S, Sharma J. Screening of urinary isolates for the prevalence and antimicrobial susceptibility of enterobacteria other than E.coli. International Journal of Life Science and Pharma Research 2013;3(1):L-100-4.

[27] Shaifali I, Gupta U, Mahmood SE, et al. Antibiotic susceptibility patterns of urinary pathogens in female outpatients. N Am J Med Sci 2012;4(4):163-9.

[28] Tambekar DH, Dhanokar DV, Gulhane SR, et al. Antibacterial susceptibility of some urinary tract pathogens to commonly used antibiotics. Afr J Biotechnol 2006;5(17):1562-5.

[29] Karlowsky JA, Kelly LJ, Thornsberry C, et al. Trends in antimicrobial resistance among urinary tract infection isolates of E.coli from female outpatients in United States. Antimicrob Agents Chemother 2002;46(8):2540-5.

[30] Gales AC, Jones RN, Turnidge J, et al. Characterization of pseudomonas aeruginosa isolates: occurrence rates, antimicrobial susceptibility patterns and molecular typing in the global SENETRY antimicrobial surveillance program 1997-1999. Clin Infect Dis 2001;32(Suppl 2):S146-55.

[31] Tankhiwale SS, Jalgaonkar SV, Ahmad S, et al. Evaluation of extended spectrum beat lactamase in urinary isolates. Indian J Med Res 2004;120(6):553-6.

[32] Amin M, Mehdinejad M, Pourdangchi Z. Study of bacteria isolated from urinary tract infections and determination of their susceptibility to antibiotics. Jundishapur Journal of Microbiology 2009;2(3):11823.

[33] Gururajan G, Kathireshan AK, Ramasamy B. Prevalence of extended spectrum beta lactamases in uropathogenic escherichia coli and klebsiella species in a Chennai suburban tertiary care hospital and its antibiogram pattern. Res J Microbiol 2011;6(11):796804.

[34] Maya AS, Prabhakar K, Hanna LE, et al. Phenotypic and genotypic characterisation of extended spectrum beta lactamse producing escherichia coli clinical isolates from semi urban area. J Pharm Res 2011;4(1):6-10.
[35] Dogra V, Sharma A, Mishra B, et al. Drug resistance gram neg bacilli in UTI: a need for strict antibiotic prescription policy. Int Journal of Health \& Allied Sciences 2012;1(3):204-6.

[36] Tantry BA, Rahiman S. Antibacterial resistance \& trend of urinary tract pathogens to commonly used antibiotics in Kashmir Valley. West Indian Med J 2012;61(7):703-7.

[37] Eshwarappa M, Dosegowda R, Aprameya IV, et al. Clinico-microbiological profile of urinary tract infection in south India. Indian $\mathrm{J}$ Nephrol 2011;21(1):30-6.

[38] Kothari A, Sagar V. Antibiotic resistance in pathogens causing community acquired UTI in India: a multicentre study. J Infect Dev Ctries 2008;2(5):354-8.

[39] Farajnia S, Alikhani MY, Ghotaslou R, et al. Causative agents and antimicrobial susceptibility of urinary tract infections in the northwest of Iran. Int J Infect Dis 2009;13(2):140-4.

[40] Goldstein FW. Antibiotic susceptibility of bacterial strains isolated from patients with community acquired urinary tract infections in France. Multicentre study group. Eur J Clin Microbiol Infect Dis 2000;19(2):112-7.

[41] Orrett FA, Davis GK. A comparison of antimicrobial susceptibility profile of urinary pathogens for the years, 1999 and 2003. West Indian Med J 2006;55(2):95-9.

[42] Gupta V, Yadav A, Joshi RM. Antibiotic resistance pattern in uropathogens. Indian J Med Microbiol 2002;20(2):96-8.

[43] Uwaezuoke JC, Ogbulie JN. Antibiotic sensitivity pattern of urinary tract pathogens in Port-Harcort, Nigeria. J Appl Sci Environm Mange 2006;10(3):103-7.

[44] Choudhari P, Chaudhari SN, Chattopadhyay C. Frequency of UTI in children and antibacterial resistance pattern of urinary isolates in India. International Journal of Pharmaceutical Science and Healthcare 2013;2(3):37-41.

[45] Bora A, Ahmed GU, Hazarika NK. Phenotypic detection of ext. Sp. B. Lact. and Amp C beta lactamase in urinary isolates of E. coli at a tertiary care referral hospital in northeast India. Journal of College of Medical SciencesNepal 2012;8(3):22-8.

[46] Shiju MP, Yashavnath R, Narendra N. Detection of extended spectrum beta-lactamase production and multidrug resistance in clinical isolates of E.coli and K. pneumoniae in Mangalore. J Clin Diag Res 2010;4(3):2442-5.

[47] Jadhav S, Hussain A, Devi S, et al. Virulence characteristics and genetic affinities of multiple drug resistant uropathogenic escherichia coli from a semi urban locality in India. PLoS One 2011;6(3):el8063.

[48] Akata F, Tatman-Otkum M, Ozkan E, et al. Prevalence of extended spectrum beta lactamases produced by nosocomial isolates of enterobacteriaceae in Trakta University Hospital, Turkey, New Microbiol 2003;26:257-62.

[49] Gales AC, Sader HS, Jones RN. SENTRY participants group (Latin America). Urinary tract infection's trends in the American hospitals: reports from the SENTRY antimicrobial surveillance programme (1997-2000). Diagn Microbial Infect Dis 2002;44:289-99. 
[50] Mathur P, Kapil A, Das B, et al. Prevalence of extended spectrum beta lactamase producing gram negative bacteria in a tertiary care hospital. Indian J Med Res 2002;115:153-7.

[51] Subha A, Ananthan S. Extended spectrum beta lactamases (ESBL) mediated resistance to third generation cephalosporins among klebsiella pneumonia in Chennai. Indian J Med Microbiol 2002;20(2):92-5.
[52] Ryan KJ. Urinary tract infections. In: Ryan KJ, Ray CG, (eds). Sherris Medical Microbiology. New York: McGraw-Hill 2004:867-71.

[53] Duttatroy B, Mehta S. Extended spectrum beta lactamses (ESBL) in clinical isolates of klebsiella pneumonia and escherichia coli. Indian $\mathrm{J}$ Pathol Microbiol 2005;48(1):45-8. 